\title{
Collective beam instability study using a correlation-moment analysis
}

\author{
Samuel A Heifets and Alexander W. Chao \\ Stanford Linear Accelerator Center, Stanford University, Stanford, California 94309, USA
}

(Received 20 October 2006; published 29 March 2007)

\begin{abstract}
A general formalism for treating simultaneously the transverse coupled-bunch and transverse coupledmode instabilities is presented. In this approach, the equations of motion of a coupled multibunch beam are expanded to yield a system of equations involving correlation moments of the transverse and longitudinal motions. After a proper truncation, the system of equations is closed and can be solved. This approach allows us to formulate within one framework several known instability mechanisms including the single-bunch mode-coupling instability, the coupled-bunch instability, the mode-coupling instability, and the coupled-mode coupled-bunch instability as particular cases.
\end{abstract}

DOI: 10.1103/PhysRevSTAB.10.034402

PACS numbers: 29.27.Bd

\section{INTRODUCTION}

In a storage ring, a train of bunches is subject to various collective instabilities if the beam current is sufficiently high. For a single-bunch beam, instabilities are traditionally analyzed by decomposing the collective motion of the particles in the bunch into collective modes; the instability then results from the coupling among these modes. This is referred to as the mode-coupling instability in the literature. Another mechanism deals with a train of multiple bunches; the corresponding instabilities can be analyzed in terms of the coupled motion among the interacting bunches while basically ignoring the internal degrees of freedom within each individual bunch. This is referred to as coupled-bunch instability in the literature. Traditional treatment of collective instabilities in storage rings considers the mode-coupling instability and the coupled-bunch instability separately [1]. An exception is the coupledmode coupled-bunch (CMCB) instability [2] where the effect of bunch coupling is taken into account in description of the mode-coupling instability. In the present paper, we develop an alternative formalism of collective instabilities where these two types of instabilities are treated systematically within one framework.

One traditional way of analyzing the beam stability is based on the linearized Vlasov equation $[1,2]$. The formalism we present here adopts an alternative approach, based on expanding the equations of motion into a system of equations for correlation moments, to be defined later, of the transverse and longitudinal degrees of freedom. After a proper truncation, the system of equations is closed and can be solved. Because of the process of truncation, our approach is limited to the lowest few modes, while the modes higher than the quadrupole modes are ignored. However, the latter have higher thresholds and, usually, are less important.

We will limit ourselves to the transverse collective instabilities, even though these instabilities necessarily involve the longitudinal beam dynamics as well.

\section{EQUATION OF MOTION}

Let us consider a train of $n_{b}$ bunches in a storage ring with circumference $C=2 \pi R$ and the revolution period $T_{0}=C / c$. For simplicity, we assume the uniform fill of the ring with the equidistant bunches separated by $s_{B}=c \tau_{b}$. In the absence of collective motion, the center of the leading bunch in the train is at the location $s=c t$ around the ring at time $t$. We consider the ultrarelativistic case when particles move with the speed of light $c$. The center of the $N$ th bunch is at the distance $s_{N}=(N-1) s_{B}>0$ behind the (arbitrary chosen for the uniform fill) leading bunch for evenly spaced bunches. The variable $s(-\infty<$ $s<\infty)$, rather than the time $t$, is chosen as the independent variable because the properties of the focusing system are defined by their location $s$. Position $s_{i, N}(t)$ of the $i$ th particle in the $N$ th bunch, $N=1,2, \ldots, n_{b}$, is

$$
s_{i, N}(t)=c t-s_{N}+z_{i, N}(s),
$$

where $z_{i, N}$ is longitudinal displacement due to synchrotron motion with a sign convention that $z_{i, N}>0$ indicates the displacement is toward the head of the bunch.

We want to study the effects of the coupling of the longitudinal and transverse oscillations assuming that the bunch current is below the threshold of the longitudinal microwave instability [3]. The longitudinal distribution of particles in this case is a steady-state Haissinski distribution [4], and the single-particle trajectory gives

$$
z_{i, N}(s)=\zeta_{N}(s)+a_{i, N} \sin \left(\omega_{s} s / c+\phi_{i, N}\right),
$$

where $\zeta_{N}$ describes the motion of the bunch centroid. We assume below that $\zeta_{N}=0$ neglecting the longitudinal coupled-bunch effect although the consideration can be easily generalized to take it into account. Generally, the trajectory $z_{i, N}(s)$ in the nonlinear steady-state Haissinski potential has the harmonics multiple of the synchrotron frequency $\omega_{s}$. However, the strong head-tail instability due to the mode coupling is, usually, caused by the crossings of the lowest two monopolar and dipolar modes. Therefore, it 
is sufficient to consider only the lowest harmonics of the synchrotron frequency $\omega_{s}$ but taking into account correlation of the longitudinal and transverse motion in the same bunch. Our approach allows us to consider the strong and chromatic head-tail instabilities simultaneously.

Collective instabilities are caused by the wakefields generated by the beam at irregularities of the vacuum chamber. If $t$ is the time when the $i$ th particle in the $N$ th bunch is at the position $s_{i, N}(t)$ of the vacuum system, the time $t_{j, M, k}$ when a particle $j$ of the $M$ th bunch is at the same location on the turn number $k(-\infty<k<\infty)$ is

$$
t_{j, M, k}=t_{M, k}^{0}+\frac{1}{c}\left[z_{i, N}(s)-z_{j, M}(s-k C)\right],
$$

where

$$
t_{M, k}^{0}=t-k T_{0}+(M-N) \tau_{b} .
$$

The transverse offset of the $i$ th particle in the $N$ th bunch around the ring is

$$
y_{i, N}(s)=A_{i, N}(s) e^{-i \psi_{i, N}(s)}+\text { c.c. },
$$

where the transverse phase advance $\psi_{i, N}$ and the betatron frequency $\omega_{i, N}^{y}$ are

$$
\psi_{i, N}(s)=\frac{\omega_{y} s}{c}-\omega_{\xi} \frac{z_{i, N}(s)}{c}, \quad \frac{\omega_{i, N}^{y}}{c}=\frac{d \psi_{i, N}}{d s} .
$$

Here, $\omega_{y}$ is the nominal betatron frequency, and [5]

$$
\omega_{\xi}=\frac{\xi}{\alpha} \omega_{y}, \quad \xi=\frac{1}{\omega_{y}} \frac{d \omega_{y}}{d \delta}
$$

are the chromatic head-tail frequency and the relative chromaticity, respectively; $\alpha$ is the momentum compaction factor, and $\delta=\Delta E / E$ is the relative energy shift of a particle. In general, the amplitude $A_{i, N}$ is complex and is slowly varying in time.

The equation of motion in the smooth focusing approximation is

$$
\begin{aligned}
\frac{d^{2} y_{i, N}(s)}{d s^{2}}+\left(\frac{\omega_{i, N}^{y}}{c}\right)^{2} y_{i, N}(s)= & \frac{r_{e}}{\gamma C} \sum_{j, M, k} W_{y}\left(t-t_{j, M, k}\right) \\
& \times y_{j, M}(s-k C),
\end{aligned}
$$

where $r_{e}$ is the classical radius of the particle, and $\gamma$ is the relativistic factor. The right-hand side (RHS) is due to the wakefields and is responsible for the collective instabilities; $W_{y}(t)$ is the transverse wake per turn (dimension $\mathrm{V} / \mathrm{pC} / \mathrm{m}$ or $1 / \mathrm{cm}^{2}$ ). For an ultrarelativistic beam, causality requires that $W_{y}(t)=0$ for $t<0$. Summation over $k$ takes into account long-range wakefields that last a multiple number of revolutions.

Usually, the coherent frequency shift and the instability growth rate are small compared to $\omega_{y}$. In this case, Eq. (8) can be averaged over fast oscillations, giving the equation for the amplitudes,

$$
\begin{aligned}
\frac{d A_{i, N}(s)}{d s}= & i \lambda_{y} \sum_{j, M, k} W_{y}\left(t-t_{j, M, k}\right) A_{j, M}(s-k C) \\
& \times e^{i k \omega_{y} T_{0}-i\left(\omega_{\xi} / c\right)\left[z_{i, N}(s)-z_{j, M}(s-k C)\right]},
\end{aligned}
$$

where we have dropped terms $d^{2} A_{i, N} / d s^{2}$ and $d \omega_{i, N}^{y} / d s$, and introduced

$$
\lambda_{y}=\frac{r_{e} c}{2 \gamma C \omega_{y}}
$$

\section{CORRELATION-MOMENT EXPANSION}

Measurements of the beam dynamics, usually, detect only the bunch centroid motion. We want to reduce Eq. (9) to the system of equations for the quantities averaged over $N_{b}$ particles in a bunch

$$
\overline{A_{M}(s)}=\frac{1}{N_{b}} \sum_{i=1}^{N_{b}} A_{i, M}(s) .
$$

Equation (9) shows that the transverse and longitudinal motions are correlated. In this paper, we consider transverse coherent effects assuming that the longitudinal motion is uncorrelated and can be described by Eq. (2). In this way we neglect the longitudinal coherent effects although the suggested formalism can be generalized to include this effect into account.

The averaging denoted in Eq. (11) by the bar means averaging over the full 2D distribution function depending on the transverse and longitudinal coordinates. We assume that the correlation is weak and only the lowest order correlation moments need to be taken into account. We postulate that the $2 \mathrm{D}$ averages in this case can be expanded over the low correlation moments $\left\langle A_{M}\right\rangle,\left\langle A_{M} z_{M}\right\rangle$, etc., which will be denoted by the angular brackets. For example,

$$
\begin{aligned}
\overline{A_{M} z_{M}^{l}}= & \left\langle A_{M}\right\rangle\left\langle\left\langle z_{M}^{l}\right\rangle\right\rangle+\left\langle A_{M} z_{M}\right\rangle\left\langle\left\langle z_{M}^{l-1}\right\rangle\right\rangle \frac{l !}{1 !(l-1) !} \\
& +\left\langle A_{M} z_{M}^{2}\right\rangle\left\langle\left\langle z_{M}^{l-2}\right\rangle\right\rangle \frac{l !}{2 !(l-2) !}+\cdots,
\end{aligned}
$$

where the double angular brackets mean averaging over the longitudinal uncorrelated motion. The latter can be carried out by integrating with the longitudinal distribution function $\rho_{M}\left(z, p_{z}, s\right)$ of the $M$ th bunch, for example,

$$
\left\langle\left\langle z_{M}^{l-1}\right\rangle\right\rangle=\int d z d p_{z} \rho_{M}\left(z, p_{z}, s\right) z^{l-1} .
$$

The correlation moments $\left\langle A_{M}\right\rangle,\left\langle A_{M} z_{M}\right\rangle$, etc. introduced in Eq. (12) are unknown and satisfy equations derived below.

Expansion Eq. (12) is quite analogous to the expansion of a multiparticle distribution function in the theory of statistical mechanics [6] in series over the product of single-particle distribution functions plus terms which 
take into account two-, three-, etc. particle correlations. The correlation moments introduced in Eq. (12) have simple meaning. We show that the lowest $\left\langle A_{M}\right\rangle$ describes the dipole transverse oscillations of a rigid bunch and its grows reflects the dipole coherent instability. The moments $\left\langle A_{M} z_{M}\right\rangle$ and $\left\langle A_{M} z_{M}^{2}\right\rangle$ describe correlation of the transverse motion with the dipole and quadrupolar longitudinal moments, respectively, and are responsible for the higher order transverse coherent instabilities. Because the growth rate of higher order instabilities goes down with the order, we can expect the hierarchy of correlation moments. The validity of our approach is demonstrated in this paper by reproducing results on well-known transverse instabilities [1]. We show also that the new formalism may have some advantages.

Expanding over correlation moments, we assume that there is no correlation of the longitudinal and transverse motion of particles belonging to different bunches, i.e. $\left\langle z_{i M} z_{j N}\right\rangle=0,\left\langle z_{i M} A_{j N}\right\rangle=0$ if $N \neq M$, and neglect higher order correlation moments such as $\left\langle z_{i M} z_{j N}^{2}\right\rangle$. These assumptions do not preclude bunch-to-bunch coupling of the amplitudes $\left\langle A_{M}(s)\right\rangle$ of different bunches. Using these assumptions, we can reduce Eq. (9) to a system of equations for the lowest order correlation moments.
It is convenient to write Eq. (9) in terms of the transverse impedance per turn $Z_{y}$,

$$
\begin{aligned}
\frac{d A_{i, N}(s)}{d s}= & \frac{1}{N_{b}} \sum_{k, M, j} \int \frac{d \omega}{2 \pi} F_{k}(\omega, N-M) \\
& \times A_{j, M}(s-k C) e^{i \kappa\left[z_{i, N}(s)-z_{j, M}(s-k C)\right]},
\end{aligned}
$$

where $\kappa=\left(\omega-\omega_{\xi}\right) / c$, and

$$
F_{k}(\omega, N-M)=-\lambda_{y} N_{b} Z_{y}(\omega) e^{-i\left(\omega-\omega_{y}\right) k T_{0}-i \omega \tau_{b}(N-M)}
$$

The equation for $d\left\langle A_{M}(s)\right\rangle / d s$ can be obtained from Eq. (14) using definition Eq. (11).

Expanding exponents in the RHS in series over $z_{i, N}$ and $z_{j, M}$,

$$
\begin{aligned}
e^{i \kappa\left[z_{i, N}(s)-z_{j, M}(s-k C)\right]}= & \sum_{l=0}^{\infty} \frac{i^{l}}{l !} \kappa^{l}\left[z_{i, N}(s)\right]^{l} \sum_{l^{\prime}=0}^{\infty} \frac{(-i)^{l^{\prime}}}{l^{\prime} !} \\
& \times \kappa^{l^{\prime}}\left[z_{j, M}(s-k C)\right]^{l^{\prime}}
\end{aligned}
$$

and calculating the average using Eq. (12), we get

$$
\overline{A_{j, M}(s-k C) e^{i \kappa\left[z_{i, N}(s)-z_{j, M}(s-k C)\right]}}=|G(\kappa)|^{2}\left\{A_{M}^{0}(s-k C)-\frac{\kappa}{2}\left[Q_{M}^{+}(s-k C) e^{i\left(\omega_{s} / c\right)(s-k C)}-Q_{M}^{-}(s-k C) e^{-i\left(\omega_{s} / c\right)(s-k C)}\right]\right\} .
$$

Here we have used Eq. (2) for $z_{N}(s)$ and notations

$$
A_{M}^{0}(s)=\left\langle A_{M}(s)\right\rangle, \quad Q_{M}^{ \pm}(s)=\left\langle a_{M}(s) e^{ \pm i \phi_{M}} A_{M}(s)\right\rangle, \quad G(\kappa)=\left\langle\left\langle e^{-i \kappa z_{N}}\right\rangle\right\rangle .
$$

Because the time dependence of a trajectory $z_{M}(s)$ can be considered as a canonical transform preserving the phase volume, the average $\left\langle\left\langle z_{M}^{l-1}\right\rangle\right\rangle$ for the steady-state distribution $\rho\left(z, p_{z}\right)$ is time independent. If all bunches have the same longitudinal profile, then $G$ is also independent of the bunch number. For the Gaussian distribution with the rms bunch length $\sigma, G(\Delta \omega)$ is easy to calculate,

$$
G\left(\frac{\Delta \Omega}{c}\right)=\int \frac{d z d p}{2 \pi \sigma \delta} e^{-\left[p^{2} /\left(2 \delta^{2}\right)\right]-\left[z^{2} /\left(2 \sigma^{2}\right)\right]} e^{-i[(\Delta \Omega) / c] a \sin \left(\omega_{s} s / c+\phi\right)}=e^{-(1 / 2)[(\Delta \Omega \sigma) / c]^{2}} .
$$

Equation (14) after averaging takes the form

$$
\frac{d A_{N}^{0}(s)}{d s}=\sum_{k, M} \int \frac{d \omega}{2 \pi} F_{k}(\omega, N-M)|G(\kappa)|^{2}\left\{A_{M}^{0}(s-k C)-\frac{\kappa}{2}\left[Q_{M}^{+}(s-k C) e^{i\left(\omega_{s} / c\right)(s-k C)}-Q_{M}^{-}(s-k C) e^{-i\left(\omega_{s} / c\right)(s-k C)}\right]\right\} .
$$

Similarly, multiplying Eq. (14) by $z_{i, N}\left(s^{\prime}\right)$ and calculating the sum over $i$, we get a new equation. The left-hand side (LHS) takes the form

$$
\frac{d}{d s} \frac{1}{N_{b}} \sum_{i} A_{i, N}(s) z_{i, N}\left(s^{\prime}\right)=\frac{1}{2 i} \frac{d}{d s}\left\{Q_{N}^{+}(s) e^{i \omega_{s} s^{\prime} / c}-Q_{N}^{-}(s) e^{-i \omega_{s} s^{\prime} / c}\right\}
$$

The average of the RHS can be calculated in the same way as above using the following result for the average: 


$$
\left\langle\left\langle z_{N}\left(s^{\prime}\right) e^{i \kappa z_{N}(s)}\right\rangle\right\rangle=-i \frac{\partial}{\partial \kappa} G^{*}(\kappa) \cos \left[\frac{\omega_{s}}{c}\left(s-s^{\prime}\right)\right] .
$$

Separating terms proportional to $e^{ \pm i \omega_{s} s^{\prime} / c}$, we get two equations

$$
\begin{aligned}
\pm \frac{1}{2 i} \frac{d Q_{N}^{ \pm}(s)}{d s}= & -\frac{i}{2} \sum_{k, M} \int \frac{d \omega}{2 \pi} F_{k}(\omega, N-M) G(\kappa) \frac{d G^{*}(\kappa)}{d \kappa} e^{\mp i \omega_{s} s / c} \\
& \times\left\{A_{M}^{0}(s-k C)-\frac{\kappa}{2}\left[Q_{M}^{+}(s-k C) e^{i\left(\omega_{s} / c\right)(s-k C)}-Q_{M}^{-}(s-k C) e^{-i\left(\omega_{s} / c\right)(s-k C)}\right]\right\} .
\end{aligned}
$$

It is convenient to write Eqs. (20) and (23) in the frequency domain for the Fourier components $\tilde{A}_{M}^{0}(\Omega)$ and $\tilde{Q}^{ \pm}(\Omega)$,

$$
A_{M}^{0}(s)=\int \frac{d \Omega}{2 \pi} \tilde{A}_{M}^{0}(\Omega) e^{-i[(\Omega s) / c]}, \quad Q_{M}^{ \pm}(s)=\int \frac{d \Omega}{2 \pi} \tilde{Q}_{M}^{ \pm}(\Omega) e^{-i[(\Omega s) / c]} .
$$

Fourier harmonics $\tilde{A}_{N}^{0}(\Omega)$ satisfy the following equation:

$$
\Omega \tilde{A}_{N}^{0}(\Omega)=i c \sum_{k, M} \int \frac{d \omega}{2 \pi} F_{k}(\omega, N-M)|G(\kappa)|^{2} e^{i \Omega k T_{0}}\left\{\tilde{A}_{M}^{0}(\Omega)-\frac{\kappa}{2}\left[\tilde{Q}_{M}^{+}\left(\Omega+\omega_{s}\right)-\tilde{Q}_{M}^{-}\left(\Omega-\omega_{s}\right)\right]\right\} .
$$

The sum over turns $k$ can be calculated using

$$
\sum_{k} e^{i \nu T_{0} k}=\omega_{0} \sum_{k} \delta\left[\nu+k \omega_{0}\right]
$$

where $\delta(x)$ is the $\delta$-function. Hence,

$$
\Omega \tilde{A}_{N}^{0}(\Omega)=-i \frac{\lambda_{y} \omega_{0} c N_{b}}{2 \pi} \sum_{M, k} Z_{y}(\omega)|G(\kappa)|^{2} e^{-i \omega \tau_{b}(N-M)}\left\{\tilde{A}_{M}^{0}(\Omega)-\frac{\kappa}{2}\left[Q_{M}^{+}\left(\Omega+\omega_{s}\right)-\tilde{Q}_{M}^{-}\left(\Omega-\omega_{s}\right)\right]\right\},
$$

where $\omega=\omega_{y}+\Omega+k \omega_{0}$.

Similarly, for harmonics $\tilde{Q}^{ \pm}(\Omega)$ we get

$$
\begin{aligned}
& \left(\Omega+\omega_{s}\right) \tilde{Q}_{N}^{+}\left(\Omega+\omega_{s}\right)=-i \frac{\lambda_{y} \omega_{0} c N_{b}}{2 \pi} \sum_{M, k} Z_{y}(\omega) G(\kappa) \frac{\partial G^{*}(\kappa)}{\partial \kappa} e^{-i \omega \tau_{b}(N-M)}\left\{\tilde{A}_{M}^{0}(\Omega)-\frac{\kappa}{2}\left[Q_{M}^{+}\left(\Omega+\omega_{s}\right)-\tilde{Q}_{M}^{-}\left(\Omega-\omega_{s}\right)\right]\right\} \\
& \left(\Omega-\omega_{s}\right) \tilde{Q}_{N}^{-}\left(\Omega-\omega_{s}\right)=i \frac{\lambda_{y} \omega_{0} c N_{b}}{2 \pi} \sum_{M, k} Z_{y}(\omega) G(\kappa) \frac{\partial G^{*}(\kappa)}{\partial \kappa} e^{-i \omega \tau_{b}(N-M)}\left\{\tilde{A}_{M}^{0}(\Omega)-\frac{\kappa}{2}\left[Q_{M}^{+}\left(\Omega+\omega_{s}\right)-\tilde{Q}_{M}^{-}\left(\Omega-\omega_{s}\right)\right]\right\}
\end{aligned}
$$

where again $\omega=\omega_{y}+\Omega+k \omega_{0}$.

\section{A. Eigenmode expansion}

The coupled-bunch motion can be analyzed by expanding oscillations of individual bunches over the eigenmodes. For the uniform distribution of $n_{b}$ bunches in the ring, the normalized eigenmodes are

$$
\begin{gathered}
X_{M}^{\mu}=\frac{1}{\sqrt{n_{b}}} e^{\left[(2 \pi i) / n_{b}\right](M-1) \mu}, \quad M=1,2, \ldots, n_{b}, \\
\mu=0,1, \ldots, n_{b}-1 .
\end{gathered}
$$

The form of the expansion over eigenmodes is defined by the condition of periodicity which can be explained by the following arguments. The average of the transverse offset of the $M$ th bunch

$$
y_{j, M}(s)=A_{j, M}(s) e^{-(i / c)\left[\omega_{y} s-\omega_{\xi} z_{j, M}(s)\right]}+\text { c.c. }
$$

takes the form

$$
\begin{aligned}
\left\langle y_{M}(s)\right\rangle= & {\left[A_{M}^{0}(s)+\frac{\omega_{\xi}}{2 c}\left[Q_{M}^{+}(s) e^{\left[\left(i \omega_{s}\right) / c\right] s}\right.\right.} \\
& \left.\left.-Q_{M}^{-}(s) e^{\left[\left(i \omega_{s}\right) / c\right] s}\right]\right] G^{*}\left(\omega_{\xi}\right) e^{-i\left[\left(\omega_{y} s\right) / c\right]}+\text { c.c. }
\end{aligned}
$$

For a fixed time $t$, moving from a bunch number $M$ to $M+$ $n_{b}$ brings us to the same bunch while $s=c t-(M-1) s_{b}$ changes to $s-C$. Therefore, the transverse offset of the $M$ th bunch has to satisfy the condition of periodicity,

$$
\left\langle y_{M+n_{b}}(s-C)\right\rangle=\left\langle y_{M}(s)\right\rangle .
$$

Hence, the periodicity conditions for the amplitudes are 


$$
\begin{aligned}
A_{M+n_{b}}^{0}(s-C) e^{i \omega_{y} T_{0}} & =A_{M}^{0}(s), \\
Q_{M}^{ \pm}+N_{b} & (s-C) e^{i\left(\omega_{y} \mp \omega\right) T_{0}}=Q_{M}^{ \pm}(s) .
\end{aligned}
$$

For Fourier amplitudes the conditions take the form

$$
\begin{aligned}
& \tilde{A}_{N+n_{b}}^{0}(\Omega) e^{i\left(\Omega+\omega_{y}\right) T_{0}}=\tilde{A}_{N}^{0}(\Omega), \\
& \tilde{Q}_{\bar{M}+n_{b}}^{ \pm}(\Omega) e^{i\left(\Omega+\omega_{y} \mp \omega_{s}\right) T_{0}}=\tilde{Q}_{\bar{M}}^{ \pm}(\Omega) .
\end{aligned}
$$

Equation (34) defines the phase factor in the expansion over the eigenmodes,

$$
\begin{aligned}
& \tilde{A}_{M}^{0}(\Omega)=e^{-i\left(\omega_{y}+\Omega\right) \tau_{b}(M-1)} \sum_{\mu} g_{\mu}^{0}(\Omega) X_{M}^{\mu}, \\
& \tilde{Q}_{M}^{ \pm}(\Omega)=e^{-i\left(\omega_{y}+\Omega \mp \omega_{s}\right) \tau_{b}(M-1)} \sum_{\mu} g_{\mu}^{ \pm}(\Omega) X_{M}^{\mu} .
\end{aligned}
$$

Substituting Eq. (35) into Eq. (27) and using orthogonality of the eigenfunctions $X_{M}^{\mu}$, we get

$$
\begin{aligned}
\Omega g_{\mu}^{0}(\Omega)= & -i \frac{\lambda_{y} \omega_{0} c N_{b}}{2 \pi} \sum_{N, M, \nu, k}\left[X_{N}^{\mu}\right]^{*} X_{M}^{\nu} e^{i k \omega_{0} \tau_{b}(N-M)} \\
& \times Z_{y}\left(\omega_{y}^{0}+\Omega+k \omega_{0}\right)|G(\kappa)|^{2} \\
& \times\left\{g_{\nu}^{0}(\Omega)-\frac{\kappa}{2}\left[g_{\nu}^{+}\left(\Omega+\omega_{s}\right)-g_{\nu}^{-}\left(\Omega-\omega_{s}\right)\right]\right\},
\end{aligned}
$$

where $\kappa=\left(\omega_{y}^{0}+\Omega+k \omega_{0}-\omega_{\xi}\right) / c$. Summing over $N, M$ gives

$$
\sum_{N, M}\left[X_{N}^{\mu}\right]^{*} X_{M}^{\nu} e^{i k \omega_{0} \tau_{b}(N-M)}=n_{b} \delta_{\mu, \nu} \sum_{p} \delta_{k, p n_{b}+\mu} .
$$

Equation (36) is then simplified to

$$
\begin{aligned}
\Omega g_{\mu}^{0}(\Omega)= & -i \frac{\lambda_{y} \omega_{0} c N_{b} n_{b}}{2 \pi} \sum_{p} Z_{y}\left(\omega_{p}\right)\left|G\left(\kappa_{\mu}\right)\right|^{2} \\
& \times\left\{g_{\mu}^{0}(\Omega)-\frac{\kappa_{\mu}}{2}\left[g_{\mu}^{+}\left(\Omega+\omega_{s}\right)\right.\right. \\
& \left.\left.-g_{\mu}^{-}\left(\Omega-\omega_{s}\right)\right]\right\},
\end{aligned}
$$

where

$$
\omega_{p}=\omega_{y}^{0}+\Omega+\left(p n_{b}+\mu\right) \omega_{0}, \quad \kappa_{\mu}=\frac{\omega_{p}-\omega_{\xi}}{c} .
$$

Similarly, Eq. (28) is transformed to

$$
\begin{aligned}
\left(\Omega \pm \omega_{s}\right) g_{\mu}^{ \pm}\left(\Omega \pm \omega_{s}\right)= & \mp i \frac{\lambda_{y} \omega_{0} c N_{b} n_{b}}{2 \pi} \sum_{p} Z_{y}\left[\omega_{p}\right] \\
& \times G\left(\kappa_{\mu}\right) \frac{\partial G^{*}\left(\kappa_{\mu}\right)}{\partial \kappa_{\mu}}\left\{g_{\mu}^{0}(\Omega)\right. \\
& -\frac{\kappa_{\mu}}{2}\left[g_{\mu}^{+}\left(\Omega+\omega_{s}\right)\right. \\
& \left.\left.-g_{\mu}^{-}\left(\Omega-\omega_{s}\right)\right]\right\} .
\end{aligned}
$$

Equations (38) and (40) are the system of linear equations for the amplitudes $g_{\mu}^{0}(\Omega)$, and $g_{\mu}^{ \pm}\left(\Omega \pm \omega_{s}\right)$. For Gaussian longitudinal distribution function with the $\mathrm{rms}$ bunch length $\sigma$,

$$
\begin{aligned}
\left|G\left(\kappa_{\mu}\right)\right|^{2} & =e^{-\left(\kappa_{\mu} \sigma\right)^{2}}, \\
G\left(\kappa_{\mu}\right) \frac{\partial G^{*}\left(\kappa_{\mu}\right)}{\partial \kappa_{\mu}} & =-\kappa_{\mu} \sigma^{2} e^{-\left(\kappa_{\mu} \sigma\right)^{2}} .
\end{aligned}
$$

The bunch-by-bunch feedback system adds damping to each bunch proportional to the bunch centroid velocity $\left\langle\dot{y}_{N}\right\rangle=\left(1 / N_{b}\right) \sum_{i} d y_{i, N} / d t$. The action of the feedback can be described by replacing $d^{2} y_{i, N} / d t^{2}+\left(\omega_{i, N}^{y}\right)^{2} y_{i, N}$ in the equation of motion by $d^{2} y_{i, N} / d t^{2}+2 \gamma_{F B}\left\langle d y_{N} / d t\right\rangle+$ $\left(\omega_{i, N}^{y}\right)^{2} y_{i, N}$. Equations (38) and (40) are then modified by changing the factor $\Omega$ to $\Omega+2 i \gamma_{F B}$ on the LHS.

In the following four sections, we apply the results of analysis obtained above to reproduce the well-known results for the transverse dipole coupled bunch (dipole CB) [7] of the rigid bunches and the transverse head-tail coupled-bunch instabilities. Using the same set of equations, we also obtain results for the strong and chromatic head-tail [5] instabilities of a single bunch and for the CMCB [2] instability.

\section{TRANSVERSE DIPOLE COUPLED-BUNCH INSTABILITY}

In this section, let us consider only Eq. (38) neglecting terms involving $g_{\mu}^{( \pm)}$which, as we will see later, are related to the head-tail transverse CB modes. That leaves us with a single homogeneous equation,

$$
\Omega g_{\mu}^{0}(\Omega)=-i \frac{\lambda_{y} \omega_{0} c N_{b} n_{b}}{2 \pi} \sum_{p} Z_{y}\left(\omega_{p}\right)\left|G\left(\kappa_{\mu}\right)\right|^{2} g_{\mu}^{0}(\Omega),
$$

which in turn yields the dispersion equation for the frequency $\Omega_{\mu}$ of the $\mu$ th transverse dipole CB mode,

$$
\Omega_{\mu}=-i \frac{\lambda_{y} \omega_{0} c N_{b} n_{b}}{2 \pi} \sum_{p} Z_{y}\left(\omega_{p}\right)\left|G\left(\kappa_{\mu}\right)\right|^{2} .
$$

The real part of $\Omega_{\mu}$ gives the frequency shift of the coherent mode, while its imaginary part gives the instability growth rate of the mode. If $\Omega$ is small, we can neglect it in the argument of the impedance and obtain the approximate but explicit solution of the dispersion equation. For a Gaussian bunch that gives

$$
\begin{aligned}
\Omega_{\mu}= & -i \frac{\lambda_{y} \omega_{0} c}{2 \pi} N_{B} n_{b} \sum_{p=-\infty}^{\infty} Z_{y}\left[\left(p n_{b}+\mu\right) \omega_{0}+\omega_{y}\right] \\
& \times e^{-(\sigma / c)^{2}\left[\left(p n_{b}+\mu\right) \omega_{0}+\omega_{y}-\omega_{\xi}\right]^{2}} .
\end{aligned}
$$

Note that one can relate this to the dc beam current $I_{\text {beam }}^{\mathrm{dc}}$, the beam energy $E$, and nominal betatron tune $\nu_{y}$ by 


$$
\frac{\lambda_{y} \omega_{0}}{2 \pi} N_{b} n_{b}=\frac{I_{\text {beam }}^{\mathrm{dc}}}{4 \pi(E / e) \nu_{y}} .
$$

Also note that it would be more accurate to replace the tune $\nu_{y}$ in the last formula by $R / \beta_{y}$ with the $\beta$-function taken at the location of the impedance-generating element, and note that Eq. (44) agrees with the result [8].

\section{TRANSVERSE HEAD-TAIL COUPLED-BUNCH INSTABILITY}

Here we consider Eq. (40) neglecting for a while the reverse effect of the amplitudes $g^{( \pm)}$on $g^{(0)}$, and considering the latter as an external excitation. That gives us the system of two coupled equations. Using notations

$$
\hat{g}^{ \pm}=g_{\mu}^{ \pm}\left(\Omega \pm \omega_{s}\right),
$$

where $\omega_{p}$ and $\kappa$ (we drop the index $\mu$ ) are defined in Eq. (39), and

$$
\begin{aligned}
& h_{\mu}(l)=i \frac{\lambda_{y} \omega_{0} c N_{b} n_{b}}{2 \pi} \sum_{p} Z_{y}\left[\omega_{p}\right]|G(\kappa)|^{2}\left(\frac{\kappa}{2}\right)^{l}, \\
& h_{\mu}^{\prime}(l)=i \frac{\lambda_{y} \omega_{0} c N_{b} n_{b}}{2 \pi} \sum_{p} Z_{y}\left[\omega_{p}\right] G\left(\kappa_{\mu}\right) \frac{\partial G^{*}\left(\kappa_{\mu}\right)}{\partial \kappa_{\mu}}\left(\frac{\kappa}{2}\right)^{l},
\end{aligned}
$$

we get

$$
\begin{aligned}
& {\left[\Omega+\omega_{s}-h_{\mu}^{\prime}(1)\right] \hat{g}^{+}+h_{\mu}^{\prime}(1) \hat{g}^{-}=-h_{\mu}^{\prime}(0) g_{\mu}^{0}(\Omega),} \\
& h_{\mu}^{\prime}(1) \hat{g}^{+}+\left[\Omega-\omega_{s}-h_{\mu}^{\prime}(1)\right] \hat{g}^{-}=h_{\mu}^{\prime}(0) g_{\mu}^{0}(\Omega) .
\end{aligned}
$$

The response to the excitation by the bunch centroid is infinite at the eigenfrequencies $\Omega$ given by the zeros of the determinant

$$
\operatorname{det}\left[\begin{array}{cc}
\Omega+\omega_{s}-h_{\mu}^{\prime}(1) & h_{\mu}^{\prime}(1) \\
h_{\mu}^{\prime}(1) & \Omega-\omega_{s}-h_{\mu}^{\prime}(1)
\end{array}\right]=0 .
$$

Equation (49) shows that $|\Omega| \simeq \omega_{s}$. The approximate solution for a moderate current where $\left|\kappa_{\mu}^{(2)}\right| \ll 1$ is

$$
\Omega_{\mu}^{( \pm)}= \pm \omega_{s}+h_{\mu}^{\prime}(1) .
$$

For a Gaussian bunch,

$$
\begin{aligned}
\Omega_{\mu}^{( \pm)}= & \pm \omega_{s}-i \frac{\lambda_{y} \omega_{0}}{4 \pi} N_{B} n_{b} \sum_{p=-\infty}^{\infty}\left(\frac{\sigma}{c}\right)^{2} \\
& \times\left[\left(p n_{b}+\mu\right) \omega_{0}+\omega_{y}-\omega_{\xi} \pm \omega_{s}\right]^{2} \\
& \times Z_{y}\left[\left(p n_{b}+\mu\right) \omega_{0}+\omega_{y} \pm \omega_{s}\right] \\
& \times e^{-(\sigma / c)^{2}\left[\left(p n_{b}+\mu\right) \omega_{0}+\omega_{y}-\omega_{\xi} \pm \omega_{s}\right]^{2}} .
\end{aligned}
$$

The result gives the coherent frequency shift and the growth rate of the $\mathrm{CB}$ head-tail modes.

Equation (48) defines the structure of the excited eigenmodes,

$$
\begin{aligned}
& \hat{g}^{(+)} \simeq-\frac{h_{\mu}^{\prime}(1)}{2 \omega_{s}} \hat{g}^{(-)}, \quad \Omega \simeq \omega_{\mathrm{s}} \\
& \hat{g}^{(-)} \simeq \frac{h_{\mu}^{\prime}(1)}{2 \omega_{s}} \hat{g}^{(+)}, \quad \Omega \simeq-\omega_{\mathrm{s}} .
\end{aligned}
$$

The amplitudes $\hat{g}^{ \pm}$describe correlation of the transverse and longitudinal oscillations (so-called head-tail modes). Let us consider the case of zero chromaticity, $\omega_{\xi}=0$. The correlated transverse/longitudinal motion for two head-tail modes with $\Omega \simeq \pm \omega_{s}$ is described by the terms

$$
\left\langle z_{N}(s) y_{N}(s)\right\rangle \propto \pm \frac{1}{2 i} X_{N}^{\mu} e^{-i \omega_{y} s / c-i\left(\omega_{y} \pm \omega_{s}\right) \tau_{b}(N-1)} \hat{g}_{\mu}^{ \pm}+\text {c.c. }
$$

corresponding to the periodic tilts with frequency $\omega_{s}$ of the bunch in the moving frame of the bunch centroid.

\section{MODE COUPLING IN MULTIBUNCH SYSTEM (CMCB INSTABILITY)}

Now we can take into account the effect of the head-tail modes $g_{\mu}^{( \pm)}$on the motion of the bunch centroid. The full system of Eqs. (38) and (40) is the system of linear equations $M(\Omega) V=0$ for the vector $V=\left\{g_{\mu}^{(0)}(\Omega), g_{\mu}^{(+)}(\Omega+\right.$ $\left.\left.\omega_{s}\right), g_{\mu}^{(-)}\left(\Omega-\omega_{s}\right)\right\}$. The system has a nontrivial solution at frequencies $\Omega$ given by the zeros of the determinant of the matrix $M(\Omega)$,

$$
M(\Omega)=\left[\begin{array}{ccc}
\Omega+h_{\mu}(0) & -h_{\mu}(1) & h_{\mu}(1) \\
h_{\mu}^{\prime}(0) & \Omega+\omega_{s}-h_{\mu}^{\prime}(1) & h_{\mu}^{\prime}(1) \\
-h_{\mu}^{\prime}(0) & h_{\mu}^{\prime}(1) & \Omega-\omega_{s}-h_{\mu}^{\prime}(1)
\end{array}\right]
$$

A nontrivial situation arises when the coherent tune shift is of the order of $\omega_{s}$. In this case [2], each headtail mode of a single bunch is split into an array of $n_{b}$ coupled-bunch coupled modes (CBCM) with different frequency shifts. The crossing of the $\mathrm{CBCM}$ modes takes place at different currents reducing, generally speaking, the threshold of the instability compared to that of the single-bunch CM instability. In this case, the equations cannot be considered separately as done in the previous sections and the full system has to be used to describe the CMCB instability in the multibunch system. 


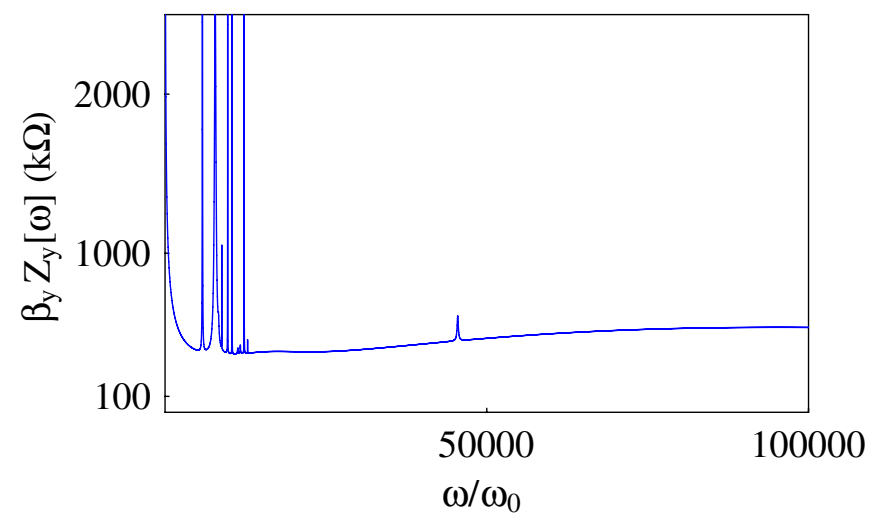

FIG. 1. (Color) Impedance of the PEP-II LER given by the contributions from 6 cavities, resistive wall, and mostly inductive vacuum components.

For illustration, we considered the PEP-II Low Energy Ring impedance shown in Fig. 1. Figure 2 shows comparison of the calculations of the strongest $\mathrm{CB}$ modes and the CMCB modes using Eqs. (44) and (54), respectively, for the beam current $I_{\text {beam }}^{\mathrm{dc}}=1 \mathrm{~A}$ and the number of bunches $n_{b}=1616$. The maximum growth rate for rigid-dipole CB modes is $0.751 / \mathrm{ms}$, and for dipole CMCB modes is 0.88
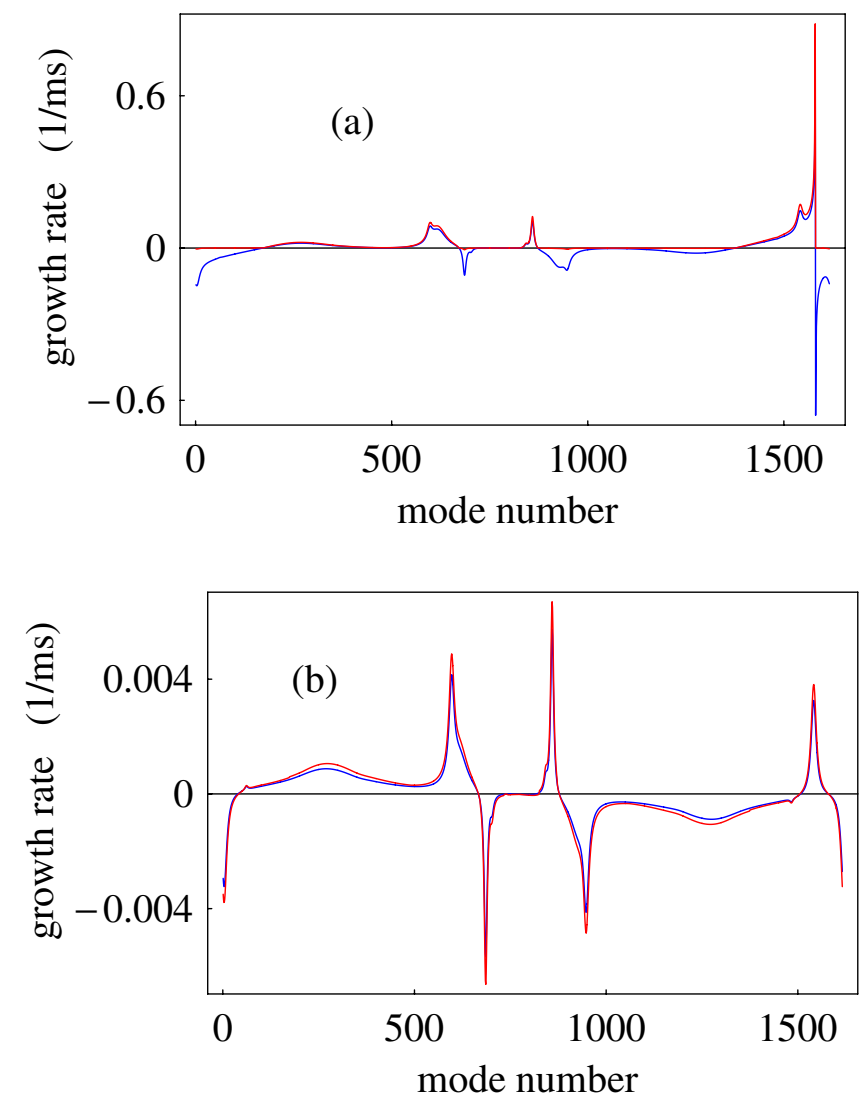

FIG. 2. (Color) The maximum growth rate of all for CB (blue) and CMCB modes (red) for the rigid-dipole modes $m=0$ (a) and head-tail $m=1$ modes (b).
$1 / \mathrm{ms}$. For the head-tail modes, the growth rates for $\mathrm{CB}$ and $\mathrm{CMCB}$ modes are $0.00571 / \mathrm{ms}$ and $0.00671 / \mathrm{ms}$, respectively.

As Fig. 2 shows, the mode coupling in this example gives only a small correction to the coupled-bunch instability because for the parameters and the impedance of the PEP-II LER the threshold of the single mode-coupling instability is much higher than the actual bunch current.

The big difference in the thresholds of the $\mathrm{CM}$ and CBCM instabilities can be expected close to the threshold of the CM instability provided the long-range wake produces sufficiently large frequency spread of the CB modes.
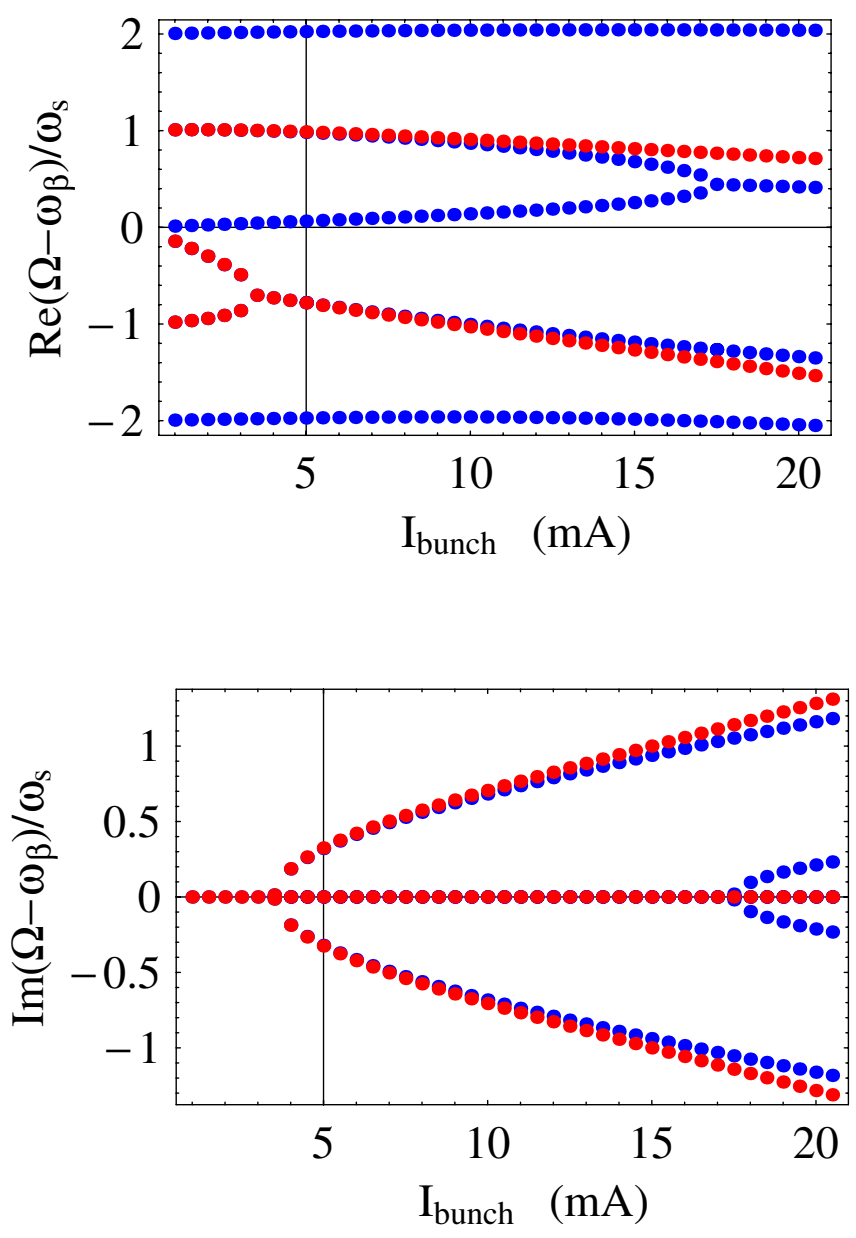

FIG. 3. (Color) The growth rate vs bunch current for the sum of the broadband and the resistive wall impedances. Top window: the maximum growth rate of all coupled modes of a single bunch calculated with the Satoh-Chin formalism (red dots) and using Eq. (56) (blue line). Agreement is quite good. Bottom window: comparison of the growth rate of the single-bunch head-tail instability (blue line, the same as in the top window) and the maximum growth rate of all modes of the CBCM instability for 1600 bunches (red line) calculated using Eq. (54). Dependence on the bunch current is modified by the effect of the CB modes. For comparison, the dashed line shows the maximum growth rate of CB modes for the same impedance and 1600 bunches. The chromaticity in all cases $\xi=0$. 
We considered the case of the impedance given as the sum of the broadband and the resistive wall impedances. The latter generates large tune spread of the CB modes. The beam parameters and parameters of the broadband impedance are described in Appendix A. The $\mathrm{Cu}$ conductivity and the beam pipe radius $b=3 \mathrm{~cm}$ were used for the standard form of the resistive wall impedance. Results are shown in Fig. 3. Agreement of our theory with the SatohChin formalism is again very good. The result in the bottom window demonstrates the CBCM effect: dependence of the growth rate on the bunch current for a single bunch and for 1600 uniformly distributed bunches are substantially different.

\section{HEAD-TAIL INSTABILITY}

Let us apply these results to a single bunch, putting $n_{b}=$ $N=M=1, \mu=0$ in Eqs. (27) and (28). Let us use notations

$$
\begin{aligned}
\hat{A}^{0} & =\tilde{A}_{N}^{0}(\Omega), \\
\hat{Q}^{ \pm} & =\tilde{Q}^{( \pm)}\left(\Omega \pm \omega_{s}\right), \\
h(l) & =i \frac{\lambda_{y} \omega_{0} c N_{b}}{2 \pi} \sum_{k} Z_{y}\left[\omega_{k}\right]|G(\kappa)|^{2}\left(\frac{\kappa}{2}\right)^{l}, \\
h^{\prime}(l) & =i \frac{\lambda_{y} \omega_{0} c N_{b}}{2 \pi} \sum_{k} Z_{y}\left[\omega_{k}\right] G(\kappa) \frac{\partial G^{*}(\kappa)}{\partial \kappa}\left(\frac{\kappa}{2}\right)^{l},
\end{aligned}
$$

where $\omega_{k}=\omega_{y}+k \omega_{0}+\Omega, \kappa=\left(\omega_{k}-\omega_{\xi}\right) / c$. The system of linear equations takes the form

$$
\begin{aligned}
\Omega \hat{A}^{0} & =-h(0) \hat{A}^{0}+h(1)\left[\hat{Q}^{+}-\hat{Q}^{-}\right], \\
\left(\Omega+\omega_{s}\right) \hat{Q}^{+} & =-h^{\prime}(0) \hat{A}^{0}+h^{\prime}(1)\left[\hat{Q}^{+}-\hat{Q}^{-}\right], \\
\left(\Omega-\omega_{s}\right) \hat{Q}^{-} & =h^{\prime}(0) \hat{A}^{0}-h^{\prime}(1)\left[\hat{Q}^{+}-\hat{Q}^{-}\right] .
\end{aligned}
$$

Solutions exist if

$\operatorname{det}\left[\begin{array}{ccc}\Omega+h(0) & -h(1) & h(1) \\ h^{\prime}(0) & \Omega+\omega_{s}-h^{\prime}(1) & h^{\prime}(1) \\ -h^{\prime}(0) & h^{\prime}(1) & \Omega-\omega_{s}-h^{\prime}(1)\end{array}\right]=0$.

In the lowest order in the beam current, the roots are

$$
\Omega=-h(0), \quad \Omega= \pm \omega_{s}+h^{\prime}(1) .
$$

The result Eq. (58) includes both strong and chromatic head-tail effects.

We can compare this result with the Satoh-Chin theory of the head-tail instability for a Gaussian bunch (which also includes both strong and chromatic head-tail effects), see Appendix A. The Satoh-Chin theory gives the solution in terms of an infinite matrix $M_{h, l},(h, l)=0,1,2, \ldots$ To get the solution, the matrix is truncated to finite rank. The rank of the matrix defines how many synchrotron modes are taken into account. The threshold, usually, corresponds to the crossing of the modes with mode indices $m=0$ and $m=-1$. Therefore, it can be defined with a good accuracy truncating the matrix to the rank $r=1$, taking only components $(h, l)=0$ and $(h, l)=1$. In this approximation and in the lowest order in the bunch current, the roots of the Satoh-Chin theory are

$$
\Omega=-i K_{\mathrm{SCh}} \omega_{s} M_{0,0}, \quad \Omega= \pm \omega_{s}-i K_{\mathrm{SCh}} \omega_{s} M_{1,1} .
$$

The Satoh-Chin coefficient $K_{\mathrm{SCh}}$ is

$$
K_{\mathrm{SCh}}=\frac{\lambda_{y} \omega_{0} N_{b} c}{2 \pi \omega_{s}}
$$

and the matrix $M_{h, l}(\lambda)$ in the Satoh-Chin formalism gives

$$
h(0)=i K_{\mathrm{SCh}} \omega_{s} M_{0,0}, \quad h^{\prime}(1)=-i K_{\mathrm{SCh}} \omega_{s} M_{1,1} \text {. }
$$

Therefore, the roots, Eq. (59), are exactly the same as given by the Satoh-Chin theory in this approximation. We also compared the result of Eq. (57) numerically with the Satoh-Chin formalism with the rank of the truncated matrix equal to 2. The result is shown in Fig. 4. Parameters are the same as in [9], see Appendix A. The chromaticity has been set to $\xi=0$. The threshold of instability is defined by the crossings of the modes $m=0$ and $m=-1$. Agreement of the results is quite good and confirms that the higher synchrotron modes give only a small correction to the threshold.

The advantage of our approach is that Eq. (57) does not require the Gaussian bunch profile, thus allowing us to take into account the potential well distortion (PWD). Figure 5 shows the threshold of the strong head-tail instability for a Gaussian bunch (blue dots) and for the bunch with profile

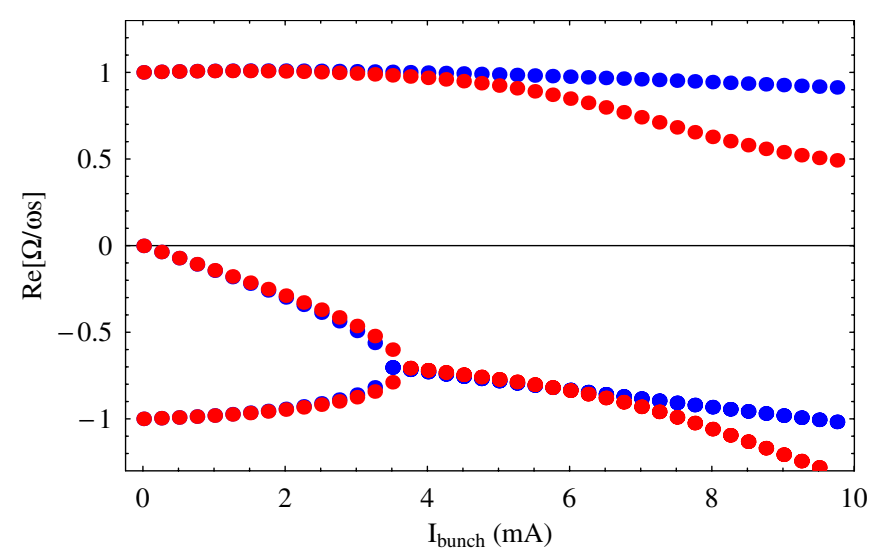

FIG. 4. (Color) The strong head-tail instability for a Gaussian bunch, the broadband $Q=1$ impedance, and the zero chromaticity. The blue dots are calculated using the Satoh-Chin formalism where the synchrobetatron modes $m=0, \pm 1, \pm 2$ are taken into account. The threshold of instability is defined by the crossings of the modes $m=0$ and $m=-1$. The red dots show the result of calculations based on Eq. (57) for the same Gaussian bunch. The threshold in the last case is in good agreement with the more accurate first method. 

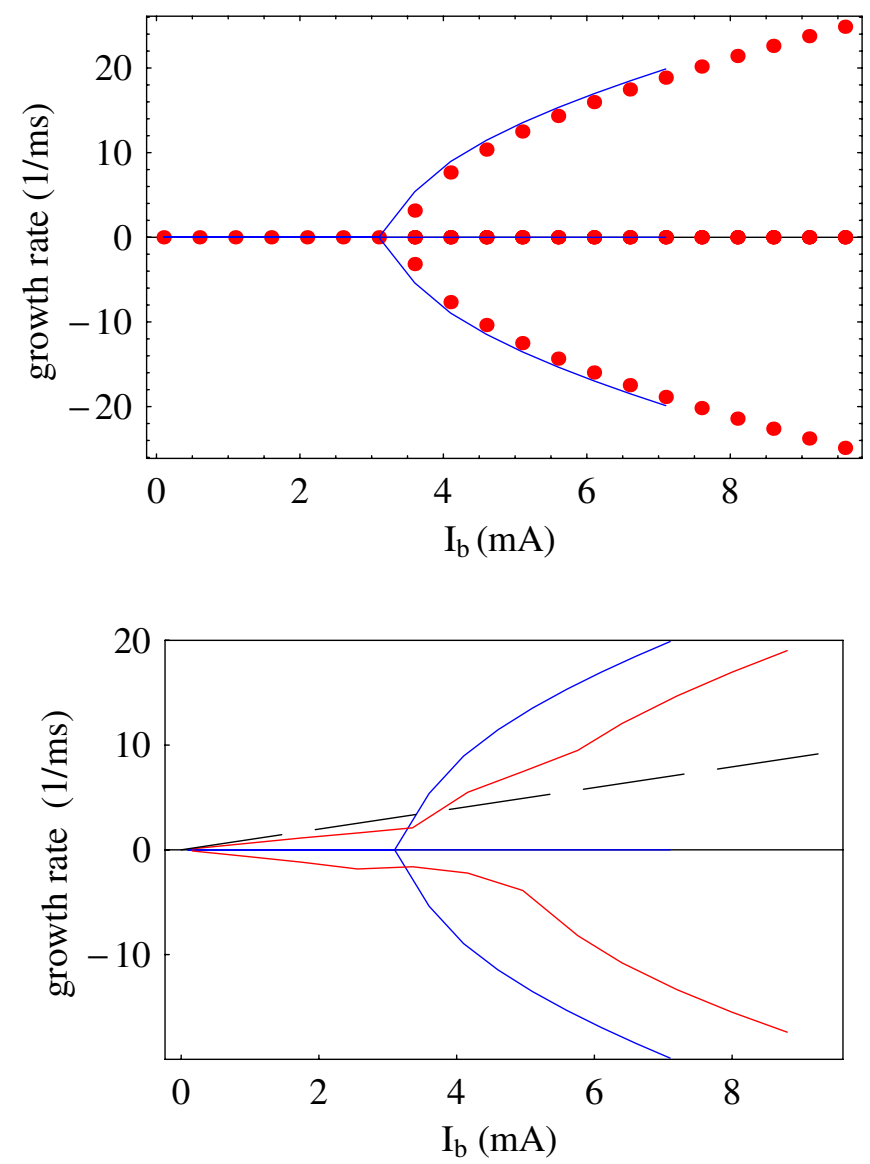

FIG. 5. (Color) The mode-coupling instability for a single-bunch calculated for the broadband $Q=1$ impedance and the zero chromaticity. The blue dots are calculated using the Satoh-Chin formalism for a Gaussian bunch, where the synchrobetatron modes $m=0, \pm 1, \pm 2$ are taken into account (the rank of the matrix $r=2$ ). The red dots show the result of calculations based on Eq. (57) taking into account the potential well distortion. The thresholds in both cases are practically the same, although the eigenmode frequencies differ at higher bunch currents.

given by the current dependent Haissinski distribution. The effect on the threshold is negligible. More accurately, to be consistent, taking into account the PWD we should also take into account the synchrotron frequency spread $\omega_{s}(a)$ calculating the $G(\kappa)$ factor. Such generalization of our formalism does not contradict to single harmonic approximation Eq. (2).

\section{SUMMARY}

The transverse instabilities of coupled bunches are studied considering correlation of the transverse and longitudinal motion. We show that the formalism allows obtaining the results for dipole and head-tail coupledbunch instabilities, the single-bunch coupled-mode instability, as well as the results for the coupled-bunch coupledmode instability. All of that can be obtained as limiting cases of the same framework of equations. Applying this method for a single bunch gives results which agree quite well with the Satoh-Chin theory of the head-tail instability for a Gaussian bunch. The equations derived here for the head-tail instability allow us to take into account also the potential wake distortion of the bunch profile. The statements are illustrated by numerical examples. We believe that the correlation-moment analysis presented here provides a way to describe the collective beam instability mechanism as an alternative, and in some aspects an improvement, compared with the usual analysis based on linearization of the Vlasov equation.

\section{ACKNOWLEDGMENTS}

This work was supported by Department of Energy Contract No. DE-AC03-76SF00515.

\section{APPENDIX A: SATOH-CHIN FORMALISM FOR HEAD-TAIL INSTABILITY}

The accurate consideration of the head-tail instability for a Gaussian bunch was given by Satoh and Chin [9]. The result is formulated as a matrix equation

$$
\operatorname{det}\left[\delta_{h, l}+i K_{\mathrm{SCh}} b_{h}(\lambda) M_{h, l}\right]=0,
$$

for the parameter $\lambda=\Omega / \omega_{s}$, where $\Omega$ is the coherent shift from the zero-current betatron tune $\nu_{\perp}$, the instability takes place when the growth rate $\operatorname{Im}[\Omega]>0$, and

$$
K_{\mathrm{SCh}}=\frac{I_{\mathrm{bunch}} \beta_{\perp}}{4 \pi(E / e) \nu_{s}}
$$

Here $M_{h, l}, h, l=0,1,2, \ldots$ is the matrix element

$$
\begin{aligned}
M_{h, l}= & \sum_{p=-\infty}^{\infty} Z_{\perp}\left[\left(p+\nu_{\perp}+\lambda \nu_{s}\right) \omega_{0}\right] \\
& \times C_{h}\left[\left(p+\nu_{\perp}+\lambda \nu_{s}-\nu_{\perp} \frac{\xi}{\alpha}\right) \frac{\sigma}{R}\right] \\
& \times C_{l}\left[\left(p+\nu_{\perp}+\lambda \nu_{s}-\nu_{\perp} \frac{\xi}{\alpha}\right) \frac{\sigma}{R}\right],
\end{aligned}
$$

where $Z_{\perp}(\omega)$ is transverse impedance (dimension $\left.\Omega / \mathrm{m}\right) \xi$ is the relative chromaticity, $R$ is the average machine radius, $\alpha$ is the momentum compaction, and

$$
C_{h}(x)=\frac{1}{\sqrt{h !}}\left(\frac{x}{\sqrt{2}}\right)^{h} e^{-\left(x^{2} / 2\right)} .
$$

The coefficients $b_{h}(\lambda)$ are

$$
\begin{gathered}
b_{h}(\lambda)=\sum_{k=0}^{[h / 2]} \frac{h !}{k !(h-k) !} \frac{\lambda}{\lambda^{2}-(h-2 k)^{2}} P[h, k], \\
b_{0}(\lambda)=\frac{1}{\lambda}, \quad b_{1}(\lambda)=\frac{2 \lambda}{\lambda^{2}-1} .
\end{gathered}
$$

The upper limit of summation is given by the integer part of $h / 2$ and $P(h, k)=1$ if $2 k=h$ and $P(h, k)=2$ other- 
wise. In actual calculations, the matrix is truncated to a finite rank which is approximately equal to the number of azimuthal modes taken into account. Usually, the threshold of instability is given by the lowest modes. An example of calculations based on the Satoh-Chin formalism is given in the text. For illustration in the text above, we took parameters of the broadband $Q=1$ wake used by Satoh-Chin: the resonance frequency $f_{\text {res }}=1.3 \mathrm{GHz}$, the shunt impedance $R_{s}=0.68 \mathrm{M} \Omega / \mathrm{m}$, energy $E=14.5 \mathrm{GeV}$, the synchrotron tune $\nu_{s}=0.044$, the betatron tune $\nu_{y}=21.25$, the momentum compaction $\alpha=1.2 \times 10^{-3}$, the revolution frequency $f_{\text {rev }}=136.4 \mathrm{kHz}$, rms bunch length $\sigma=$ $2 \mathrm{~cm}$, and $\beta$-function $\beta_{y}=160 \mathrm{~m}$.

[1] See, for example, Alexander W. Chao, Physics of Collective Beam Instabilities in High Energy Accelerators (J.
Wiley and Sons, Inc., New York, 1993), and references therein.

[2] J. S. Berg and R. D. Ruth, Phys. Rev. E 52, R2179 (1995).

[3] E. Keil and W. Schnell, CERN Report TH-RF/69-48, 1969; D. Boussard, CERN Lab II/RF/Int 75-2, 1975.

[4] J. Haissinski, Nuovo Cimento B 18, 72 (1973).

[5] C. Pellegrini, Nuovo Cimento A 64, 447 (1969); M. Sands, SLAC Reports TN-69-8 and TN-69-10, 1969.

[6] R. Balescu, Equilibrium and Nonequilibrium Statistical Mechanics (Wiley Interscience, London-New YorkSydney, 1975).

[7] Ernest D. Courant and Andrew M. Sessler, Rev. Sci. Instrum. 37, 1579 (1966).

[8] M. S. Zisman, S. Chattopadhyay, and J. J. Bisognano, ZAP user's manual, LBL-21270, UC-28, 1986.

[9] K. Satoh and Y. Chin, Nucl. Instrum. Methods 207, 309 (1983). 